\section{Aplicaciones móviles en modelos de microemprendimientos: Métricas y fortalezas en el comercio ecuatoriano}

Mobile applications in microenterprise models: Metrics and strengths in Ecuadorian commerce

\section{Pedro Cesar Godoy Rosero* Marisol Del Rocio Alava Cedeño* Wendy Jesenia Añapa Tapuyo* Harold Stalin Quiñonez Francis*}

\section{RESUMEN}

El auge de las aplicaciones móviles en el ámbito comercial generó un impacto positivo para la economía de los negocios ecuatorianos que utilizaron en sus procesos la tecnología móvil, sin embargo, muchos emprendedores no acoplaron sus actividades a modelos de negocio innovadores. Esta investigación tiene como objetivo describir las métricas y fortalezas de la utilización de aplicaciones móviles en modelos de microemprendimientos del sector comercial ecuatoriano. Mediante un enfoque metodológico mixto, se aplicó en primera instancia un cuestionario con 0,86 de coeficiente

\footnotetext{
* Magister en Seguridad de las tecnologías de la información y de las Comunicaciones, docente investigador en la facultad de Ciencias Administrativas y Económicas de la Universidad Técnica Luis Vargas Torres de Esmeraldas. Correo electrónico cesar.godoy@utelvt.edu.ec, https://orcid.org/0000-0002-1944-780 I
}

* Magister en Administración de Empresas, docente investigadora de la Facultad de Ciencias Administrativas y Económicas de la Universidad Técnica Luis Vargas Torres de Esmeraldas. Correo electrónico marisol.alava@utelvt.edu.ec, https://orcid.org/0000-0002-5I25-2860

\footnotetext{
*Estudiante de 7mo. Semestre de la Carrera de Contabilidad y Auditoría de la Facultad de Ciencias Administrativas y Económicas de la Universidad Técnica Luis Vargas Torres de Esmeraldas, Correo electrónico wendy.anapa@utelvt.edu.ec, https://orcid.org/0000-0002-94I5-075X

* Magister en Administración de Empresas, docente investigadora de la Facultad de Ciencias Administrativas y Económicas de la Universidad Técnica Luis Vargas Torres de Esmeraldas. Correo electrónico harold.quinonez@utelvt.edu.ec, https://orcid.org/0000-0002-7537-4838
}

\section{REVISTATECNOLÓGICA ciencia y educación Edwards Deming}

ISSN: 2600-5867

\section{Atribución/Reconocimiento-NoCo} mercial- Compartirlgual 4.0 Licencia Pública Internacional — CC

\section{BY-NC-SA 4.0}

https://creativecommons.org/licenses /by-nc- sa/4.0/legalcode.es

Editado por: Tecnológico Superior Corporativo Edwards Deming Enero - Marzo Vol. 6 - I - 2022 https://revista-edwardsdeming.com/index.php/es e-ISSN: 2576-097I

Recibido: 22 julio 2021

Aprobado: 16 septiembre, 2021

Pag 50 - 63 
de validación estadística, logrando realizar la analítica de datos estadísticos a 5.763 microemprendimientos de la provincia de Esmeraldas, lo cual determinó un nivel considerable de utilización de aplicaciones digitales en el contexto comercial. Así mismo, se realizaron 25 entrevistas a emprendedores que promueven sus productos y/o servicios utilizando aplicaciones móviles. Los hallazgos del estudio determinaron que más del $58 \%$ de los microemprendimientos promocionan sus productos y/o servicios en redes sociales como Facebook, WhatsApp e Instagram, apoyándose en sistemas de delivery como Portear, Rappi, Cabify y Uber eats, los cuales permitieron la apertura de nuevas ideas de negocio según los reportes de métricas establecidas, lo se considera como una fortaleza comercial.

Palabras clave: Aplicaciones móviles, Micromprendimiento, modelos de negocio, métricas para aplicaciones.

\section{ABSTRACT}

The rise of mobile applications in the commercial field generated a positive impact on the business economy, however, many entrepreneurs did not couple their activities to innovative business models. This research aims to describe the metrics and strengths of the use of mobile applications in micro-enterprise models of the Ecuadorian commercial sector. Using a mixed methodological approach, a questionnaire with a statistical validation coefficient of 0.86 was applied in the first instance, managing to perform statistical data analysis on 5,763 micro-enterprises in the province of Esmeraldas, which determined a considerable level of use of digital applications. in the commercial context. Likewise, 25 interviews were conducted with entrepreneurs who promote their products and / or services using mobile applications. The findings of the study determined that more than $58 \%$ of microenterprises promote their products and / or services on social networks such as Facebook, WhatsApp and Instagram, relying on delivery systems such as Portear, Rappi, Cabify and Uber eats, which allowed the opening of new business ideas according to the established metric reports, it is considered as commercial strength.

Keywords: Mobile applications, Micro-understanding, business models, metrics for applications.

\section{INTRODUCCIÓN}

Los avances de la tecnología móvil y los efectos que conlleva la mueva normalidad, empezaron a reactivar la economía mundial, la visión y gestión emprendedora de los 
microemprendimientos, se apoyó en las aplicaciones móviles para innovar en sus modelos de negocios y enfrentar la problemática multifacética que modificó el comportamiento, los hábitos de consumo, los gustos y preferencias del mercado consumidor de bienes y servicios que provee el comercio en sus diversas manifestaciones.

En el Ecuador, surgió una gran variedad de microemprendimientos con oferta de servicios y productos que a pesar de una marcada crisis económica global, pudieron ingresar y mantenerse al ritmo de los negocios digitales apoyándose en aplicaciones móviles para la promoción, publicación y venta, el impacto positivo de estos nuevos modelos de negocio se evidencia con el surgimiento de actividades colaterales como las de servicio de entrega a domicilio. (Rizo-Mustelier, Miriela; Villa-Tabares, Belkis; VueltaLorenzo, Daniel Rafael; VargasBatis, Belyani, 2017).

En el marco de la actividad comercial y surgimiento de nuevos modelos de negocio, en la presente investigación se planteó como objetivo describir las métricas y fortalezas de la utilización de aplicaciones móviles en modelos de microemprendimientos del sector comercial ecuatoriano, abarcando desde un cuestionario la variable nivel de utilización de aplicaciones tecnológicas; las categorías fundamentales del estudio fueron abordadas considerando las aplicaciones móviles y los modelos de microemprendimiento.

En el proceso de transición a la nueva normalidad, los negocios digitales consideraron ciertas herramientas tecnológicas para entrar al mercado digital con el negocio ya implementado; entre estas, utilizaron redes sociales, servicios web interactivos con ecommerce y el e-mail marketing. Este giro del modelo de negocio de lo tradicional a lo digital, permitió identificar claramente las prioridades y las señales del negocio, esto sirvió de apoyo para que las empresas aprovecharan al máximo las aplicaciones móviles y las mejores prácticas disponibles en la WEB. (Zoom Empresarial , 2021).

Esta investigación, es generada desde el proyecto de vinculación Fomento innovador de modelos empresariales y gestión de la calidad a emprendimientos y mipymes en el entorno comercial, económico, social, ambiental y tecnológico de la provincia de Esmeraldas.

\section{MATERIALES Y MÉTODOS}

La presente investigación es de tipo descriptiva según lo indican (Roberto Hernández, Carlos Fernández, Pilar Baptista, 2010), se aplicó el enfoque metodológico mixto, considerando que inicialmente fue utilizado un cuestionario validado estadísticamente con 0,86 de coeficiente de Alfa de Cronbach, logrando realizar la analítica de datos estadísticos a 5.763 microemprendimientos de la provincia de Esmeraldas, así mismo, se realizaron 25 entrevistas semiestructuradas a propietarios de micro emprendimientos que promocionaron sus productos y/o servicios mediante aplicaciones móviles, la entrevista semiestructurada de 6 preguntas respondidas de forma oral, fue validada por criterio de expertos (Cienfuegos y Cienfuegos, 2016).

Tabla I. Fiabilidad estadística 


\begin{tabular}{ccc}
\hline \hline Estadística de fiabilidad & \\
\hline Alfa de Cronbach & N de elementos \\
, 86 & & 15 \\
\hline \hline
\end{tabular}

Fuente: Cuestionario sobre aplicaciones móviles en microempredimientos 2021 n=5763

Se valió del método inductivo partiendo de premisas particulares para luego inferir los resultados de la muestra hacia toda la población (Medina H., Fernando, Marco Galván, 2017). El objeto de estudio se relaciona con el análisis cuantitativo y cualitativo sobre modelos de microemprendimientos del sector comercial ecuatoriano, considerando las métricas y fortalezas de la utilización de aplicaciones móviles. El proceso metodológico abordó la variable denominada utilización de aplicaciones tecnológicas en microemprendimientos, la cual fue parte del cuestionario aplicado, obteniendo el siguiente gráfico de acuerdo con el análisis estadístico realizado.

Gráfico I. Aplicaciones tecnológicas

Utiliza aplicaciones tecnológicas para

fortalecer las ventas en su negocio

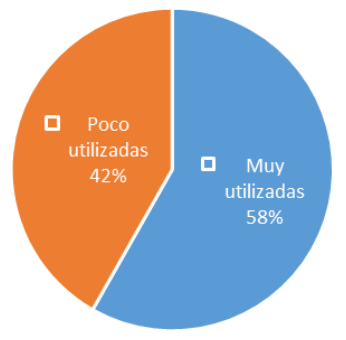

Fuente: Cuestionario sobre aplicaciones móviles en microempredimientos 2021

De acuerdo con el análisis del gráfico anterior, se procedió a seleccionar por conveniencia a 25 microemprendedores que fueron parte de la encuesta antes indicada. Se aplicó la entrevista semiestructurada con 6 preguntas, las cuales fueron validadas en función del criterio de 3 expertos conocedores de la temática de estudio, titulados con el grado de $\mathrm{PhD}$. Las respuestas orales de los entrevistados fueron sistematizadas mediante el software de análisis cuantitativo MAXQDA, mediante el cual se generaron las categorías de análisis respectivas.

El fundamento analítico y concluyente del estudio considera los antecedentes de estudio, así como a las experiencias tomadas del contexto de investigativo y sobre todo los resultados de la analítica de los datos obtenidos mediante las técnicas de investigación aplicadas.

Los emprendedores se han apoyado en el uso de dispositivos Smartphone para a través de aplicaciones hacer publicidad de los servicios y/o productos que disponen para la venta. Los sistemas operativos que soportan los dispositivos, cada usuario dispone de las tiendas de Google Play para Android, o de App Store pada dispositivos iOs en iPhone. 
Las aplicaciones móviles son programas informáticos desarrollados por programadores expertos de grandes empresas o por programadores con conocimientos básicos en estructuras programación los cuales generan líneas de código para establecer tareas específicas a los usuarios. Las aplicaciones deben ser implementadas mediante programación segura; el hosting en el que se almacene o procese información sensible, debe garantizar la utilización de protocolos de seguridad y sistemas de antivirus capaces de detener intentos de hackeo o vulnerabilidad de los sistemas implementados (José Luis Filippi, Guillermo Lafuente, Rodolfo Bertone, 2016).

Los emprendimientos están utilizando aplicaciones de redes sociales, en el caso de WhatsApp, es una aplicación utilizada en muchos países para comunicación directa con otras personas, es muy sencilla y fiable en el envío y recepción de mensajes de tipo texto o imagen, muchos emprendedores han creado un perfil de la empresa para establecer conexiones con los clientes en el lugar donde invierten su tiempo, diciendo donde encontrar el negocio (Guamán, C. R. S., Rivera, D. P. P., Vivar, S. A. M., \& Lapo, E. V. A., 202I).

En el caso de Instagram, sobre los procesos de difusión y comercio, se realizan una serie de funciones que permite comprar fácilmente a partir de las fotos y los vídeos que la marca pública en la plataforma. Fotos y videos en Instagram usa contenido en tiempo real y prediseñado para crear una experiencia de compra visual e interactiva que acerca a la audiencia. En las páginas de detalles de producto se proporciona toda la información pertinente sobre los artículos de tu catálogo, como el precio o la descripción. También enlazan al contenido de Instagram en el cual aparece etiquetado un producto y dirigen a los compradores al sitio web para procesar la transacción.

Facebook conecta con más personas de tu zona para aumentar el tráfico y las ventas en la tienda física. Permite promocionar el negocio local con Facebook actualizando sus cuentas, mostrando anuncios a las personas indicadas y perfeccionando sus campañas. (FACEBOOK, 2020).

La página empresarial de Facebook, es una herramienta gratuita que permite que las empresas, marcas, famosos, causas y organizaciones puedan llegar a su audiencia. Mientras que los perfiles de Facebook pueden ser privados, las páginas son públicas. Google puede indexar tu página para que sea más fácil de encontrar para las personas. Se puede administrar tu página de Facebook y plataformas como Facebook Business Suite y Creator Studio desde tu ordenador y tu dispositivo móvil.

Entre las ventajas de las páginas, se puede indicar que son sencillas y gratuitas: las páginas son gratis. Crear una página es cuestión de minutos, y ofrecemos muchas funciones para administrarla. Conecta con tus clientes: miles de millones de personas se comunican con empresas a través de Facebook.

Se utiliza una página para aumentar la fidelidad entre clientes nuevos y existentes. Anunciarse a través de una página con los anuncios para hacer crecer el negocio e incrementar la audiencia. Se requiere de una Fanpage o página para anunciar en Facebook, enlazando y relacionado los productos o servicios que brinde el emprendimiento con la acción de publicitar y vender, se puedes utilizar las herramientas 
publicitarias de Facebook para crear anuncios, y planear estrategias para llegar a más clientes (Espinoza, F. X. D., García, J. T. Z., Pita, G. E. C., \& Patiño, E. J. P., 2020).

Entre las principales funciones de las páginas, se puede indicar que ofrece información comercial, siendo que las páginas facilitan datos sobre tu empresa, por ejemplo, la dirección, el número de teléfono, el horario, las categorías o un nombre de usuario único. Se publica a la hora de compartir información a través de una página, lo más rápido es realizar una publicación.

El administrador de la página puede añadir un botón de llamada a la acción para incentivar a las personas a realizar acciones específicas. Así mismo, se utilizan herramientas de publicación para administrar las publicaciones programadas. Envía mensajes: las páginas incluyen una bandeja de entrada que te permite comunicarte con las personas en Facebook, Instagram y Messenger. También puedes utilizar distintas herramientas para organizar los mensajes y las respuestas automáticas (Montero Corrales, L. , 2018). En relación con las herramientas, estas tienen acceso gratis a las páginas para conectar con los clientes y ampliar el negocio. Las herramientas como "Eventos" y "Citas" pueden ayudar a hacer crecer la base de clientes, mientras que "Empleos" ayudan a encontrar candidatos cualificados.

Las insights pueden servir a encontrar clientes a los que les interese la oferta. Se visualiza con mayor claridad las acciones que se realizan en una página, entre ellas la frecuencia de activación de los comentarios positivos o negativos sobre los productos o servicios ofrecidos en el emprendimiento, lo cual se puede comentar o compartir. También se poden ver insights sobre las páginas de los competidores para aprender sobre la industria y audiencia.

Una página es una de las muchas herramientas que puedes utilizar para tener presencia en Facebook. Si se ha creado un grupo o un perfil en lugar de una página, hay que obtener más información sobre sus diferencias y sobre sus usos. Se debe tener presente que es necesario tener un perfil personal para crear una página, aunque se trate de dos recursos independientes, siendo así, se podrán diferenciar las métricas para cada página o perfil (Montero Corrales, L. , 2018).

Facebook Business Suite, un espacio único para ordenadores y dispositivos móviles diseñado con el objetivo de que las empresas accedan a las herramientas que necesitan a fin de prosperar en Facebook e Instagram. Entre las opciones que ofrece Business Suite actualmente para mejorar la experiencia de administración de una empresa en nuestras aplicaciones, se incluyen la publicación de contenido, el envío de mensajes y la posibilidad de consultar estadísticas y usar distintas funciones publicitarias.

Los estudios demuestran que a los propietarios de empresas les gustaría sacar más partido de las aplicaciones de Facebook, pero no pueden hacerlo porque deben dedicar su tiempo a las necesidades diarias de sus negocios. Business Suite les permite administrar toda su actividad desde un único lugar, lo que les da el tiempo que necesitan para centrarse en otros aspectos de la empresa.

Para sacar el máximo partido de Business Suite, es necesario empezar por vincular tus cuentas empresariales de Facebook e Instagram si todavía no lo has hecho. Esto 
permitirá aprovechar las características principales del producto y disfrutar de posibilidades como ver todas las novedades de un vistazo. Se podrá ver todas las alertas críticas, los mensajes, los comentarios y cualquier otra actividad en Facebook e Instagram que necesite la atención en la pantalla de inicio de Business Suite. Esto te permite establecer prioridades y encargarte de la actividad de tu empresa a lo largo del día. Muchas veces es necesario configurar una respuesta guardada personalizada para responder a preguntas frecuentes y crear un método abreviado para optimizar el proceso de respuesta.

Compartir con comunidades de Facebook e Instagram puedes preparar contenido para las noticias de Facebook e Instagram y programar su publicación a la hora que mejor encaje con la audiencia principal. Comprender qué funciona en la pestaña "Estadísticas", se tendrá acceso a estadísticas sobre el alcance, la interacción y el rendimiento de las publicaciones en Facebook e Instagram.

Para hacer crecer la audiencia, se puede promocionar una publicación o crear anuncios para que más personas visualicen los contenidos e interactúen con él. Para acceder a Business Suite, se debe ingresar en la cuenta de Facebook asociada a la empresa. Si se cumplen los requisitos, al acceder a business.facebook.com desde el equipo o dispositivo, se redirigirá a Business Suite. Se mostrará automáticamente la opción para empezar a usar Facebook Business Suite, si ya se utiliza la aplicación del administrador de páginas en un dispositivo móvil. Si todavía no se tiene esta aplicación, se debe descarga Facebook Business Suite desde las tiendas de aplicaciones de iOS o Android, de acuerdo al sistema operativo del equipo.

El modelo de negocio es una herramienta previa al plan de negocio, cuyo objetivo es permitir conocer con claridad el tipo de negocio que se va a crear e introducir en el mercado, a quién va dirigido, cómo se va a vender y cómo se van a conseguir los ingresos. Definir el tipo de negocio de una empresa es parte del proceso, para ello se tienen en cuentan varios parámetros (Canvas, 202I).

El parámetro económico se considera el valor financiero como una de las principales bases que se deben conocer en un modelo de negocio. Los costes, la selección de precios y la obtención de ingresos son puntos claves en el diseño de un modelo de negocio. Una empresa debe generar dinero y mantener el flujo de ingresos para que se mantenga en el mercado de manera activa un largo tiempo.

Eb relación a los componentes, las actividades, los clientes, los recursos humanos con los que contará la compañía, sus ofertas, todo ello son consideraciones que se deben plasmar en un modelo de negocio para que la empresa consiga definir en un siguiente paso sus estrategias. Las estrategias se diseñan a raíz de las investigaciones previas con el objetivo de crear un negocio competitivo y que perdure en el mercado. Con el avance de internet y las nuevas tecnologías, han aparecido multitud de modelos de negocio que anteriormente no se habían utilizado. A continuación, explicamos los modelos de negocio más comunes, (Pérez A, 2020).

El modelo de negocio tipo Franquicia se aplica en empresas con marcas de renombre que deciden conceder ciertas licencias a otros empresarios para que trabajen bajo su 
marca. En este caso, el franquiciado debe cumplir con una serie de requisitos para poder vender bajo una marca que no le pertenece. El tipo de modelo fabricación, es un modelo en el que la actividad económica consiste en producir determinados productos y venderlos, generalmente, a mayoristas que los comercialicen. Las empresas de distribución adquieren los productos producidos en las fábricas y los venden ellas mismas al consumidor final o a empresas minoristas. Ej. Supongamos el caso de la empresa que le lleva la Coca-Cola a un restaurante como modelo de negocio de distribución (Hidalgo Jaramillo, 20I7).

Las Retail, son todas aquellas empresas que venden directamente los productos que han adquirido a los distribuidores al público. En el retail podemos encontrar Walmart o Lidl, mientras que las empresas de Suscripción consisten en obtener ingresos gracias a una cuota mensual que es pagada por los clientes. Empresas como Netflix o Apple Music basan su modelo de negocio en la suscripción. Las empresas de Publicidad, son un modelo de negocio establece como fuente de ingresos principal la publicidad, es el caso de muchas páginas web. Economipedia, gracias a la publicidad puede seguir creando contenido para que se informen acerca del mundo de la economía, Google.

Los negocios de Patentes generan la investigación y el desarrollo de productos exclusivos que puedan ser patentados son la fuente de ingresos de determinadas empresas. Las empresas farmacéuticas o de piezas exclusivas de determinadas máquinas obtienen sus ingresos gracias a haber logrado una patente. Freemium, es una variante de este tipo de modelo de negocio es el freemium. Al igual que antes, aquí se ofrecen servicios o productos de forma gratuita, aunque de forma limitada. Si la persona quiere llegar a disfrutar del servicio/producto en su totalidad ya tendría que pagar (Hidalgo Jaramillo, 2017)..

Este tipo de sistema es muy habitual en el software online o en las aplicaciones para móvil. Por ejemplo, la amplia mayoría de videojuegos para móvil usan este sistema. El conocido Candy Crash o Clash of Clans son gratuitos, pero si quiere ascender en el juego más rápido debe realizar la compra del programa. Cola larga, es el modelo de negocio Long Tail se basa en una idea muy sencilla: en vez de centrar el potencial de venta en productos muy demandados (y muy competidos), se enfoca en productos con menos demanda y menos competencia. A modo de ejemplo. El encargado de una tienda de música coloca los grandes éxitos del momento en su escaparate. Todo lo que está demandando el público, él lo tiene. En cambio, sus ventas no son buenas. ¿Qué ocurre? Su tienda está en un barrio alejado del centro comercial; además allí hay varias tiendas también de música.

El modelo Cebo y Anzuelo, se refiere a ofrecer un producto a un coste muy reducido. En algunos casos se puede llegar a rebajar el precio hasta por debajo de lo que le costó a la empresa. ¿Por qué? Porque para usar ese producto, el usuario necesita comprar otro complementario que es del que vamos a obtener la rentabilidad. Esto se suele dar cuando el cebo es un producto del que no se puede sacar márgenes interesantes. En 
cambio, al reducir su precio, se aumenta la demanda. Lo que va a implicar la compra del anzuelo que es donde se obtiene una mayor rentabilidad (Hidalgo Jaramillo, 2017).

Peer to Peer, es uno de los tipos de modelos de negocios entre iguales, de consumidor a consumidor. Un ejemplo serían todas las plataformas que están surgiendo para alquilar, intercambiar o vender productos o servicios. Cada vez se ven más empresas en forma de aplicación para móvil que ponen en contacto a personas que se pueden ayudar las unas a las otras (Hidalgo Jaramillo, 2017).

En este momento los modelos de negocio más innovadores están en internet. La red se ha convertido en una gran fuente de recursos e ideas de negocio. ¿Quieres saber cuáles son los modelos de negocio online que más están funcionando? Aquí los tienes:

E-commerce: Este modelo de negocio tiene mucha relación con el del retail. En este caso, se basa en la venta online de cualquier tipo de producto. El ejemplo que se menciona también pertenece al modelo de negocio del retail pero en el mundo online. Amazon es un gran ejemplo de este modelo de negocio.

El comercio electrónico está creciendo de una forma increíble. Solo hace falta echar un vistazo a las cifras. El hecho de que la tecnología cada vez sea más accesible facilita que cualquier usuario se lance al mundo de los e-commerce. La hiper segmentación y un trato cada más especializado y personal al cliente están detrás del éxito de estos negocios.

Aproximadamente 260.000 nuevas versiones de APP o nuevas APP se crean en un mes para sistemas Android a nivel mundial, siendo las herramientas que facilitan las labores cotidianas de trabajo (Rojas, 2017). Así mismo, refiere que el objetivo de este tipo de Apps consiste en agrupar contenidos e información de diferentes restaurantes en un software, donde el consumidor realiza la toma de pedidos en la plataforma de forma rápida y fácil, y donde posteriormente incluye la entrega de este al lugar establecido.

En el ámbito local se menciona que, en el Ecuador, al menos diez aplicaciones se utilizan para solicitar comida a domicilio funcionaban, entre ellas: Domicilios.com, DeliveryEc, Menú Express, MotoDelivery, Bon Appetit App, El Café de Tere, Mandao, Domino's Pizza, UberEats o Glovo son algunas y varias multinacionales.

En la revista (Revista Líderes, 2020), se señala que en el país desde el inicio de la pandemia marcas como Rappi, Glovo, UberEats y Tipti fueron las protagonistas en lo que es la entrega de comida, medicinas y otros productos. Actualmente no se cuenta con un registro oficial que indique la cantidad exacta de las Apps de delivery que funcionan en el país. Sin embargo, luego de que el SRI incorporara en el catastro público a estas empresas como proveedoras de servicios digitales se identificaron al menos 5 plataformas que generan tributos y que se encuentran activas en el Ecuador (Diario el Comercio, 2020).

Gracias al auge del e-commerce, los servicios de delivery están aumentando de popularidad. Cada vez son más las personas que solicitan las entregas a domicilio como parte fundamental de sus compras digitales (de comida u otros productos). Sin embargo, todavía existen ciertos retos que el servicio de delivery debe afrontar para responder 
plenamente a las demandas del mercado, más aún en lo referente a las entregas a domicilio en las áreas urbanas. Antes de entrar en detalle sobre los retos del servicio de entrega a domicilio, veamos primero cómo funciona el delivery.

\section{RESULTADOS}

Tabla 2. Correlación de medias, mediante la estadística de muestras emparejadas sobre Gestión de emprendimientos y modelos de negocio

\begin{tabular}{lll}
\hline \hline & \multicolumn{1}{c}{ Correlación } & Sig. \\
\hline $\begin{array}{l}\text { App_móvil } \\
\text { (agrupado) } \\
\text { Modelo_Negocio_MEDIA }\end{array}$ &, 000 \\
\hline \hline
\end{tabular}

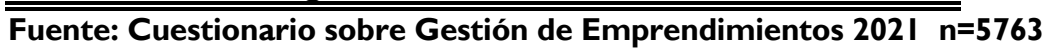

Los resultados de la prueba estadística que se observa en la Tabla 2, evidencia el valor de significancia $\mathrm{p}=0,00(3,7394 \mathrm{E}-\mathrm{I} \mathrm{I})$ correspondiente al $0 \%$ de probabilidad de fallar en el resultado de la relación existente entre las variables medidas 0,685 , considerada como moderada > a la media positiva; con lo cual se determina que la utilización de aplicaciones móviles en microemprendimientos depende del modelo de negocio aplicado en el negocio. En la prueba estadística aplicada no se consideró la relación del tiempo de vida del negocio, lo cual podría generar variabilidad en los resultados obtenidos.

Tabla 3 . Correlación de medias, mediante la estadística de muestras emparejadas sobre la utilización de aplicaciones digitales y el flujo del dinero en el emprendimiento

\begin{tabular}{ccll}
\hline \hline & $\mathrm{N}$ & Correlación & Sig. \\
\hline $\begin{array}{l}\text { Utiliza_App_Media } \\
\text { Flujo_dinero_Media }\end{array}$ & 5.500 &, 643 &, 000 \\
\hline \hline Cuestionario sobre Gestión de Emprendimientos 2021 & $n=5763$ & & \\
\hline
\end{tabular}

Cuestionario sobre Gestión de Emprendimientos 2021 n=5763

En la Tabla 3, se muestra el valor significativo de $p=0,00$ (3,34I IE-92) correspondiente al $0 \%$ de probabilidad de fallar en el resultado de la relación de las variables Utiliza_App_Media (valor medio agrupado de la utilización de aplicaciones digitales como estrategia de comercialización) y Flujo_dinero_Media (valor medio agrupado del flujo del dinero) el valor de $r=0,643$, considerada como moderada $>$ a la media positiva; con este resultado se explica que los emprendimientos que utilizan aplicaciones digitales en sus estrategias de comercialización, incide positivamente en el movimiento del flujo de dinero que obtienen los negocios. Sin embargo, se recalca que esta prueba estadística deja de lado el tipo y modelo de negocio sobre los cuales se aplicó el procedimiento estadístico.

Tabla 4. Correlación de Pearson, sobre modelos de negocio que integran las TICs en sus procesos comerciales y la implementación del teletrabajo 


\begin{tabular}{llll}
\hline \hline & & ModeloNegocioTI \\
& & Cs_Media & Teletrabajo_Media \\
\hline ModeloNegocioTICs_Media & Correlación de Pearson & $\mathrm{I}$ &, $785^{\text {** }}$ \\
& Sig. (bilateral) & &, 000 \\
& $\mathrm{~N}$ & 5763 & 5763 \\
\hline Teletrabajo_Media & Correlación de Pearson &, $785^{* *}$ & $\mathrm{I}$ \\
& Sig. (bilateral) &, 000 & \\
& $\mathrm{~N}$ & 5763 & 5763 \\
\hline \hline
\end{tabular}

Cuestionario sobre Gestión de Emprendimientos 2021 n=5763

El resultado de correlación estadística en la tabla 4, muestra que $r=0,785$ dentro del nivel de relación es considerada alta $>$ a la media positiva; de esta manera, en el marco de los modelos de negocio que utilizan las TICs en su proceso de comercialización, se resalta la relación con la aplicación de teletrabajo en los emprendimientos y MiPymes

Tabla 5. Comparación de medias, aplicando la estadística de muestras emparejadas sobre modelos de negocio con propuesta de valor y el movimiento de ventas de los emprendimientos y MyPimes.

\begin{tabular}{|c|c|c|c|c|}
\hline & & $\mathrm{N}$ & Correlación & Sig. \\
\hline $\begin{array}{l}\text { ModelosNegocio_PV } \\
\text { MovimientoVtas_MEDIA }\end{array}$ & (agrupado) & 5.500 & 897 & ,000 \\
\hline
\end{tabular}

Cuestionario sobre Gestión de Emprendimientos 2021 n=5500

En la Tabla 5, se muestra el valor sigma de $p=0,00(2,7346 E-18)$ correspondiente al $0 \%$ de probabilidad de fallar en el resultado de la relación de las variables ModelosNegocio_PV_MEDIA (valor medio agrupado) y MovimientoVtas_MEDIA, el valor de $r=0,897$, considerado como alto $>$ a la media positiva; con este resultado se explica que los modelos de negocio con propuesta de valor tiene incidencia positiva con los movimientos de ventas del objeto de estudio. En la presente prueba estadística no se ha considerado el tipo de negocio, ni el tiempo de vida de estos.

\section{DISCUSIÓN}

En relación a los aportes proporcionados cualitativamente por los emprendedores entrevistados en la ciudad de Esmeraldas, se concluye que los emprendimientos que han podido entrar al mundo de los negocios digitales, emplearon aplicaciones móviles en la WEB y en redes sociales para promocionar y posicionar sus servicios y/o productos, así como, la innovación en sus modelos de negocio, aplicaron análisis de datos mediante reportes de métricas de las aplicaciones utilizadas, tales como WhatsApp, Facebook e Instagram; de la misma manera, se apoyaron en aplicaciones vinculadas a Delivery, entre 
ellas, Portear, Rapidito, Glovo, Uber Eats y Rappi; con ello lograron la transformación de sus modelos de emprendimientos, generando un mayor crecimiento comercial en los negocios.

\section{REFERENCIAS}

Alianza para el emprendimiento e innovación. (2020). Estrategias para desarrollar un ecosistema de emprendimiento e innovación. Obtenido de https://unctad.org/system/files/official-document/epf_npd02_Ecuador_es.pdf

Barreras, I. Z. (20I8). El desarrollo tecnológico y la innovación como ente principal de competitividad en las empresas. Revista Mexicana de Agronegocios, vol. 42, 867877.

Canvas. (2021). Modelos de negocio. Nueva York: Canvas.

Catalina Nicolás Martínez, Alicia Rubio Bañón. (2020). Emprendimiento en épocas de crisis: Un análisis exploratorio de los efectos de la COVID-19. Small Business International Review. ISSN: 253 I-0046, 53-66.

Cienfuegos y Cienfuegos. (2016). Lo cuantitativo y cualitativo de la investigacion. Revista Iberoamericana para la investigacion y el desarrollo educativo, (7). 13. Recuperado el 20108/2020. ISSN: 2007-7467, , A. (2016). Un apoyo a su enseñanza . .

Diario el Comercio. (2020). Plataformas digitales. Obtenido de https://www.elcomercio.com/actualidad/negocios/aplicaciones-digitales-ivacatastro-sri.html

Diego Martín Buitrago Botero. (2020). Teletrabajo: una oportunidad en tiempos de crisis. Revista CES Derecho, 2.

Espinoza, F. X. D., García, J. T. Z., Pita, G. E. C., \& Patiño, E. J. P. (2020). La creación de fanpage en el desarrollo de emprendimientos locales. Revista San Gregorio, I(38)., 120-133.

\section{FACEBOOK. (2020). WhatsApp. FACEBOOK.}

Fernández et al. (2019). Metodología canvas como innovación estratégica para el diseño de proyectos empresariales. Revista Ciencia e Investigación, ISSN 2528-8083, Vol. 4, 87-99.

Fharitd Steewar Villegas Sabogal, Karen Lizeth Torres Perea, María Fernanda Uribe Pineda. (2020). Efectos financieros y tributarios del COVID-19 en los contribuyentes personas naturales de Villavicencio, Comuna 7. Obtenido de https://repository.ucc.edu.co/bitstream/20.500.12494/3265I/I/2020_efectos_fin ancieros_tributarios.pdf 
GEM, Global Entrepreneurship. (2016). RTR Telekom Monitor. Obtenido de https://www.rtr.at/TKP/aktuelles/publikationen/publikationen/TM_Jahresbericht -20I6.pdf

Guamán, C. R. S., Rivera, D. P. P., Vivar, S. A. M., \& Lapo, E. V. A. (202I). Transformación digital de la comercialización en las pequeñas y medianas empresas a través de redes sociales. Universidad y Sociedad, 13(3), 484-490. Revista Universidad y Sociedad, 484-489.

Isidoro Romero Luna. (2006). Las Pyme en la Economñia Global. hacia una estrategia de fomento. Problemas del desarrollo, 32-50.

José Luis Filippi, Guillermo Lafuente, Rodolfo Bertone. (2016). Aplicación móvil como instrumento de difusión. Multiciencias, 339.

Laura Angélica Décaro Santiago, M. G. (2020). Los modelos de negocios y las tecnologías de la información y comunicación. TLATEMOANI, I70-I88.

Leyva Carreras, A. B., Heredia Bustamante, J., \& Aguilar Talamante, P. (202I). El Emprendimiento como estrategia en tiempos de crisis. División De Ciencias Económicas Y Sociales, (35), I-36.

Lourdes Maribel Vásquez-Lafebre, Manuel Rafael Quevedo-Barros, Jorge Oswaldo Quevedo-Vázquez. (2020). Gerencia de Emprendimientos en Época de Pandemia. Obtenido de https://fipcaec.com/index.php/fipcaec/article/view/256

Luis Arturo Rosado Salgado, Andrés Alberto Osorio Londoño. (2020). El impacto del modelo de negocios en las capacidades dinámicas. Revista de Economía del Caribe $n^{0} .25,66-81$.

Luis Fernando Landazury Villalba, Franklin Ferrer Manotas. (2016). Innovación como eje transversal de los modelos de negocio en las organizaciones, una revisión del constructo teórico. Revista Escuela de Administración de Negocios, núm. 81, 129147.

Medina H., Fernando, Marco Galván. (2017). Imputación de datos : teoría y práctica. Obtenido de https://digitallibrary.un.org/record/6/0245?!n=es

Meléndez, M. P. (20I8). El marketing digital transforma la gestión de pymes en Colombia. Cuadernos Latinoamericanos de Administración, vol. XIV, núm. 27, I-3.

Montero Corrales, L. . (2018). Facebook y Twitter: un recorrido por las principales líneas de investigación. Revista Reflexiones, 39-52.

Nayeli Martínez Velázquez y Gabriela Dutrénit Bielous. (2019). Naturaleza de la innovación y modelo de negocios en el emprendimiento innovador. Problemas del Desarrollo. Revista Latinoamericana de Economía, vol. 50, núm. 199, 59-85. 
Organización Internacional del Trabajo. (2020). El teletrabajo durante la pandemia y después de ella. Obtenido de https://www.ilo.org/wcmsp5/groups/public/--ed_protect/---protrav/---travail/documents/publication/wcms_758007.pdf

Osterwalder y Pigneur. (2010). Business model generation: a handbook for visionaries, game changers, and Challengers. Obtenido de https://scholar.google.com.ec/scholar?q=Osterwalder+y+Pigneur+(20l0)\&hl=es \&as_sdt=0\&as_vis $=1$ \&oi=scholart

Ramón Ventura Roque Hernández, Juan Manuel Salinas Escandón, Adán López Mendoza, Juan Antonio Herrera Izaguirre. (2017). La tecnología: una herramienta de apoyo para pymes y emprendedores desde el entorno universitario. Obtenido de https://www.redalyc.org/journal// 04// 0449880008/html/

Revista Líderes. (2020). Líderes. Obtenido de https://www.revistalideres.ec/tag/lideres

Rizo-Mustelier, Miriela; Villa-Tabares, Belkis; Vuelta-Lorenzo, Daniel Rafael; VargasBatis, Belyani. (2017). Estrategias de comercialización para la gestión de ventas . Ciencia en su PC, núm. 4, 20I 7, pp. 9I-102, 3.

Roberto Hernández, Carlos Fernández, Pilar Baptista. (2010). Metodología de la investigación. Mexico: McGraw-Hill.

Rojas. (2017). Aplicaciones digitales de delivery. Obtenido de https://dspace.ups.edu.ec/bitstream/I 23456789/20087/I/UPS-GT003 I 65.pdf

Rosa M. Batista-Canino, Alicia Bolívar-Cruz, Pino Medina-Brito. (202I). Emprendimiento y Gestión Empresarial. Innovar, 83-101.

Zoom Empresarial . (202I). Negocio Digital: ¿Qué es y cómo funciona? Perú: Zoom Empresarial. 